\title{
Measurement and Analysis of Single-Hop Delay on an IP Backbone Network
}

\author{
Konstantina Papagiannaki, Student Member, IEEE, Sue Moon, Associate Member, IEEE, Chuck Fraleigh, \\ Patrick Thiran, Member, IEEE, and Christophe Diot
}

\begin{abstract}
We measure and analyze the single-hop packet delay through operational routers in the Sprint Internet protocol (IP) backbone network. After presenting our delay measurements through a single router for $\mathrm{OC}-3$ and $\mathrm{OC}-12$ link speeds, we propose a methodology to identify the factors contributing to single-hop delay. In addition to packet processing, transmission, and queueing delay at the output link, we observe the presence of very large delays that cannot be explained within the context of a first-in first-out output queue model. We isolate and analyze these outliers.

Results indicate that there is very little queueing taking place in Sprint's backbone. As link speeds increase, transmission delay decreases and the dominant part of single-hop delay is packet processing time. We show that if a packet is received and transmitted on the same linecard, it experiences less than $20 \mu$ s of delay. If the packet is transmitted across the switch fabric, its delay doubles in magnitude. We observe that processing due to IP options results in single-hop delays in the order of milliseconds. Milliseconds of delay may also be experienced by packets that do not carry IP options. We attribute those delays to router idiosyncratic behavior that affects less than $1 \%$ of the packets. Finally, we show that the queueing delay distribution is long-tailed and can be approximated with a Weibull distribution with the scale parameter $a=0.5$ and the shape parameter $b=0.6$ to 0.82 .
\end{abstract}

Index Terms-Link utilization, queueing delay, single-hop delay measurement.

\section{INTRODUCTION}

D ELAY IS A key metric in data network performance and a parameter in Internet service providers' (ISPs) service level agreements. In the Internet, packets experience delay due to transmission and propagation through the medium, as well as queueing due to cross traffic at routers. The characteristics of the traffic have significant impact on the queueing delay. Willinger $e t$ al. first reported that network traffic is self-similar rather than Poisson [1], and much research has been done since to explore the consequences of non-Poisson traffic on queueing delay. The fractional Brownian motion (FBM) model has been proposed to capture the coarse time scale behavior of network traffic, and results in queueing behavior that diverges signifi-

Manuscript received August 18, 2002; revised February 25, 2003.

K. Papagiannaki, S. Moon, and C. Diot are with Sprint ATL, Burlingame, CA 94010 USA (e-mail: dina@sprintlabs.com; sbmoon@sprintlabs.com; cdiot@sprintlabs.com).

C. Fraleigh is with NetVmg Inc., Fremont, CA 94538 USA (e-mail: cjf@netvmg.com).

P. Thiran is with the Laboratory for Computer Communications and their Applications, Faculty of Computer Sciences and Communication Systems (LCA - I\&C) - Swiss Federal Institute of Technology at Lausanne (EPFL), CH-1015 Lausanne, Switzerland (e-mail: Patrick.Thiran@epfl.ch).

Digital Object Identifier 10.1109/JSAC.2003.814410 cantly from that of the Poisson traffic model [2], [3]. Follow-up work shows that the wide-area network traffic is multifractal and exhibits varying scaling behavior depending on the time scale [4]. Recent work reveals that the queueing behavior can be approximated differently depending on the link utilization [5].

The above analyses, however, have been based on packet traces collected from a single link and fed into an output buffer, whose size and service rate vary. We are not aware of any measurement of the queueing delay on operational routers. The difficulty in measuring single-hop delay in a real network is threefold.

- Packet timestamps must be accurate enough to allow the calculation of the transit time through a router. This requires in particular that the measurement systems: 1) offer sufficient resolution to distinguish the arrival times of two consecutive packets and 2) are synchronized to an accurate global clock signal, such as global positioning system (GPS). These two conditions need to be met so that the maximum clock skew between any two measurement cards is limited enough to allow accurate calculation of the transit time of a packet from one interface to another interface of the same router.

- The amount of data easily reaches hundreds of gigabytes. Data from input and output links need to be matched to compute the time spent in the router.

- Routers have many interfaces; tapping all the input and output links to have a complete picture of the queueing behavior of any single output link is unrealistic in an operational network.

We have designed a measurement system that addresses the first two of the above difficulties, and deployed it in the Sprint tier-1 Internet protocol (IP) backbone network to collect packet traces with accurate timestamps [6]. We use optical splitters to capture and timestamp every packet traversing a link (see details in Section II). We obtain the single-hop delay of packets by computing the difference between the timestamps at the input and output monitored links. The third difficulty is not easy to overcome due to deployment cost and space issues. Although this prevents us from characterizing the queueing experienced by all packets, it does not affect the evaluation of the single-hop delays reported in this paper. In Section II, we present delay measurements of one hundred million packets matched among more than four billion packets and $400 \mathrm{~GB}$ of data collected from the Sprint IP backbone network. In Section III, we provide a methodology for the quantification of the various elements in single-hop delay. We identify the impact that: 1) transmission across the switch fabric; 2) the presence of 
TABLE I

DETAILS OF TRACES

\begin{tabular}{|c|c|c|c|c|c|c|c|c|}
\hline Set & Link & Speed & Date & Start Time & End Time & \# packets & Avg. Util. & \# matches \\
\hline \multirow{2}{*}{1} & in1 & OC-3 & Aug. 9, 2001 & $16: 56: 33$ UTC & $02: 56: 07$ UTC & $793,528,684$ & $70 \mathrm{Mbps}$ & \multirow{2}{*}{$2,781,201$} \\
\hline & out 1 & OC-3 & Aug. 9, 2001 & $16: 56: 00$ UTC & $02: 56: 07$ UTC & $567,680,718$ & $60 \mathrm{Mbps}$ & \\
\hline \multirow{2}{*}{2} & in2 & $\mathrm{OC}-3$ & Aug. 9,2001 & 16:56:03 UTC & $17: 41: 04$ UTC & $28,213,976$ & 30 Mbps & \multirow{2}{*}{$1,175,674$} \\
\hline & out2 & OC-3 & Aug. 9, 2001 & 16:56:04 UTC & 17:41:04 UTC & $48,886,948$ & $50 \mathrm{Mbps}$ & \\
\hline \multirow{2}{*}{3} & in 3 & OC-12 & Sep. 5, 2001 & 05:00:34 UTC & 11:17:11 UTC & $1,386,697,577$ & $150 \mathrm{Mbps}$ & \multirow{2}{*}{$17,613,103$} \\
\hline & out 3 & OC-12 & Sep. 5, 2001 & $05: 00: 34$ UTC & 11:17:11 UTC & $1,116,885,094$ & $250 \mathrm{Mbps}$ & \\
\hline \multirow{2}{*}{4} & in 4 & OC-12 & Sep. 5, 2001 & $05: 03: 15$ UTC & $17: 32: 50$ UTC & $157,518,386$ & $6 \mathrm{Mbps}$ & \multirow{2}{*}{$70,423,140$} \\
\hline & out 4 & OC-12 & Sep. 5, 2001 & $05: 03: 15$ UTC & $17: 32: 50$ UTC & $169,006,605$ & $6 \mathrm{Mbps}$ & \\
\hline
\end{tabular}

IP options; and 3) increased output link speed have on the delay experienced by packets through a single node. Surprisingly, in addition to the expected elements, such as transmission, queueing, and processing delays, we observe very long delays that cannot be attributed to queueing at the output link. We use a single output queue model to isolate these delays, and discuss their potential origins. Once the queueing delay component has been quantified, we analyze its tail behavior in Section IV. We summarize our findings in Section V.

\section{Delay Measurement}

We have designed passive monitoring systems that are capable of collecting and timestamping the first 44 bytes of all IP packets at link speeds up to OC-48 $(2.5 \mathrm{~Gb} / \mathrm{s})$, using the DAG card [7]. These monitoring systems have been deployed on various links in four points of presence (PoPs) of the Sprint IP backbone. We have collected day-long packet traces, and analyzed them off-line. Details about the measurement infrastructure can be found in [6].

The monitoring systems are GPS synchronized and offer a maximum clock skew of $6 \mu$ s. Details on the clock synchronization and possible errors in the accuracy of our delay measurements can be found in [8]. Consistency in the results obtained on more than 30 links, connected to different routers in all four PoP, across multiple days, gives us confidence in the accuracy of the single hop delay measurements presented in this paper.

\section{A. Collected Data}

We tap into the optical fiber and capture packets just before they enter and right after they leave a router. We denote the packet arrival time at an input link as $T_{\text {in }}$ and the packet departure at an output link, as $T_{\text {out }}$. For any given packet $n$, the single-hop delay through the router is the difference between its arrival and departure timestamps: $d(n)=T_{\text {out }}(n)-$ $T_{\text {in }}(n)$. This single-hop delay value corresponds to the total time a packet spends in a router.

Packet traces from more than 30 links, both OC-3 and OC-12, have been analyzed. In this paper, we use packet traces from four OC-3 links, collected on August 9, 2001, and four OC-12 links, collected on September 5, 2001. Those link pairs have been selected because they exhibit the highest delays observed among all our measurements. All packet traces were collected on routers of the same manufacturer and of the same architecture, running the same operating system version. We label a router's inbound link as in, and a router's outbound link as out, and refer to them as a data set for the remainder of the paper. Table I provides further details about the eight traces analyzed throughout the paper.

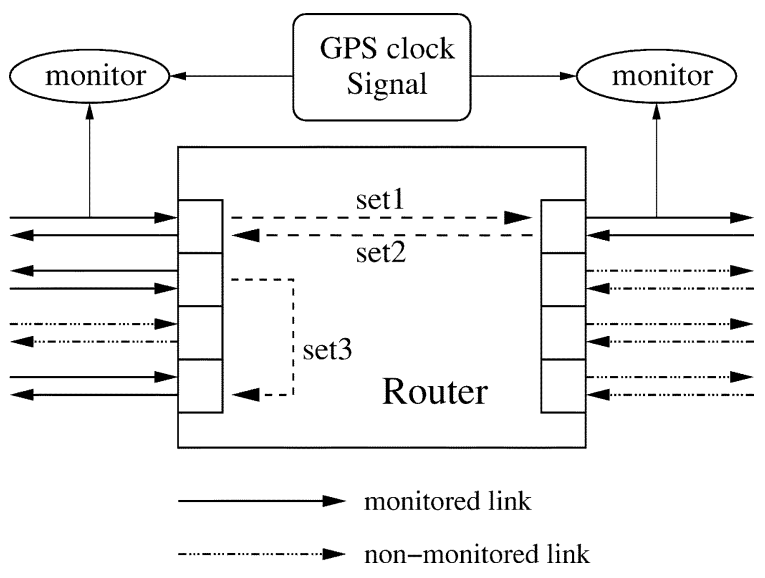

Fig. 1. Configuration of monitoring systems in a PoP.

Monitoring systems are attached to selected links inside a PoP. Seven out of the eight selected traces were collected on links attached to quad-OC-3 and quad-OC-12 linecards. Quad linecards accommodate four equal speed interfaces, as shown in Fig. 1. Packets may transit the router from one linecard to another (set1, set2), or from one interface of a linecard to another interface of the same linecard (set3).

In Table II, we present the architectural details of each data set collected. We denote each router participating in the measurements with its own index $j$. Data set1 and set2 were collected through the same router (Router1), and they correspond to the forward and reverse direction of the same router path (the incoming link of set1 is the outgoing link of set2, and vice-versa). All data sets capture the behavior of the path between two quad linecards, with the exception of set4, that corresponds to the path between a quad-OC12 card and a single OC-12 card. Data set 1 , set2, and set 4 correspond to measurements involving two different linecards, whereas set3 corresponds to measurements collected on the same linecard. In Section III-B, we show how such architectural differences affect the delay values experienced by packets through a router.

\section{B. Matching Packets}

The first step in our methodology is to identify those packets that arrive on the input links and depart on the output links we monitor. We use hashing to match packets efficiently. The hash function is based on the CRC-32 algorithm [9]. Only 30 bytes out of the 44 bytes are hashed (including the source and destination IP addresses, the IP header identification number and the whole IP header data part). The other fields are not used since they may be modified by the router (e.g., TTL) or carry almost identical information in all IP packets (e.g., IP version field, TOS byte). Using the 24 least significant bits of the 
TABLE II

Architectural Details for the Routers Where the Traces Were Collected

\begin{tabular}{c|llccc}
\hline Set & From & To & Router Name & Same Linecard & Different Linecard \\
\hline 1 & quad-OC3 & quad-OC3 & Router1 & & $\sqrt{ }$ \\
\hline 2 & quad-OC3 & quad-OC3 & Router1 & & $\sqrt{ }$ \\
\hline 3 & quad-OC12 & quad-OC12 & Router2 & $\sqrt{ }$ \\
\hline 4 & quad-OC12 & OC12 & Router3 & & $\sqrt{ }$ \\
\hline
\end{tabular}

CRC-32 value, the hash function offers an average load factor of $5.7 \%$ when one million packets are hashed into a single table. We decided to use hash tables of one million packets, because one million average-sized packets transmitted at OC-3 speeds correspond to time periods larger than one second. We assume that one second is the maximum delay a packet can experience through a single node. The hash table size is increased to four million packets for the processing of the OC-12 traces for similar reasons.

To match packets, the traces are processed as follows. The first million packets from out are hashed into a table called $H_{1}$, and the timestamp of the last packet is recorded as $e\left(H_{1}\right)$. Then, in order of arrival, each packet from in is hashed and its key value is used as an index in $H_{1}$. If table $H_{1}$ contains a packet for that specific index, we compare all 44 bytes of the two packets. If they are the same, we have a match and we output a record of all its 44 bytes, along with the timestamps for its arrival on link in and departure on link out. This process continues until we reach a packet from in that has a timestamp one second or less than $e\left(H_{1}\right)$. Then, we hash the next one million packets from out and create a second hash table $H_{2}$. Both $H_{1}$ and $H_{2}$ are used until the timestamp for a packet from in is greater than $e\left(H_{1}\right)$. When this happens, $H_{2}$ replaces $H_{1}$, and the processing continues.

Duplicate packets have been reported previously [10]. We occasionally observe them in our traces (they account for less than $0.001 \%$ of our packets), and have paid special attention to matching them. Duplicate packets have all 44 bytes identical and, therefore, hash to the same value. In most cases, we find that only after a packet left out, its duplicate arrived on in, making the classification unambiguous. We successfully match most duplicate packets with the correct arrival and departure timestamps. In other cases, we ignore the matches.

As a result of the above process, four traces of matched packets are produced. The numbers of matched packets are given in Table I. We use these traces in the next section to analyze the single-hop delay components.

\section{DELAY ANALYSIS}

We start with general observations on the delay measurements. We plot the empirical probability density function of the measured single-hop delay, and quantify step-by-step its contributing factors. The outcome of this step-by-step analysis is the derivation of the output queueing delay, which is analyzed in Section IV.

\section{A. General Observations}

We denote the $m$ th matched packet as $m$, and the total number of matched packets for a given set by $M$. Fig. 2 plots the minimum, average, and maximum values of the single-hop delay $\{d(m)\}$ across each 1 min interval for all four data sets. We observe first that the minimum delay is stable throughout all the traces, while the average delay exhibits more oscillations and may drop as the link utilization decreases toward the evening. The minimum delay corresponds to the minimum amount of time a packet needs to go through a router. Therefore, given that the minimum delay is constant throughout the day, there is at least one packet that experiences no queueing in each one minute interval.

The maximum delay is more variable than the average delay. It shows occasional spikes of a few milliseconds reaching up to $35 \mathrm{~ms}$ for set 1 and $172 \mathrm{~ms}$ for set 4 . We also note that the maximum delay remains consistently above $1 \mathrm{~ms}$ for the OC-3 data sets, and $0.2 \mathrm{~ms}$ for the $\mathrm{OC}-12$ data sets, even though the average delay decreases. We provide possible explanations in Section III-D.

\section{B. Step-by-Step Analysis of the Single-Hop Delay}

Fig. 3 presents the empirical probability density function of $\{d(m)\}, 1 \leq m \leq M$, along with various statistics on the upper right corner of each plot. Average delay values are around $100 \mu$ s for the OC-3 data sets and decrease by a factor of four when the link speed increases to OC-12. We see that $99 \%$ of the packets experience less than $1 \mathrm{~ms}$ of delay on OC-3 links. For the OC-12 traces the 99th percentile of the single-hop delay distribution is below $100 \mu$ s. However, the observed maximum delay is data set specific, reaching up to $35 \mathrm{~ms}$ in set1 and $172 \mathrm{~ms}$ in set4.

There are three distinct peaks at the beginning of each density function. Previous work by Thompson et al. reports that packets in the backbone do not have a uniform size distribution, but instead have three unique peaks at 40-44, at 552-576, and at 1500 bytes [11]. The sizes of 40 and 44 bytes correspond to minimum-sized transmission control protocol (TCP) acknowledgment packets and telnet packets of a single key stroke; 552 and 576 byte packets correspond to default maximum transmission unit (MTU) sizes when path MTU discovery is not used by a sending host; and 1500 byte packets correspond to the Ethernet MTU size. In all data sets, more than $70 \%$ of the packets are $40-$, 576-, and 1500-bytes long. We, thus, conjecture the three peaks at the beginning of the delay distribution to be related to the packet size. To verify this conjecture, we group the packets of those three sizes, and separately plot the empirical probability density function of the delay experienced by packets of the given size. Each distribution has a unique peak that matches one of the three peaks in Fig. 3. We now identify and quantify the factors that contribute the same amount of delay for packets of the same size.

1) Transmission Delay on the Output Link: A first cause is the transmission delay on the output link. Transmission delay is proportional to the packet size and to the speed of 


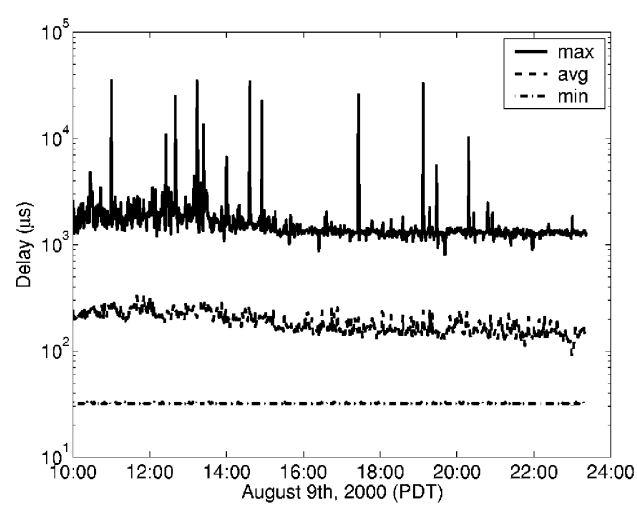

(a)

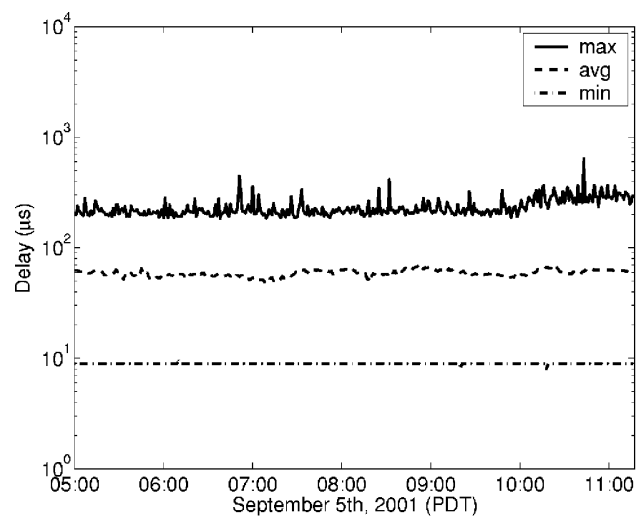

(c)

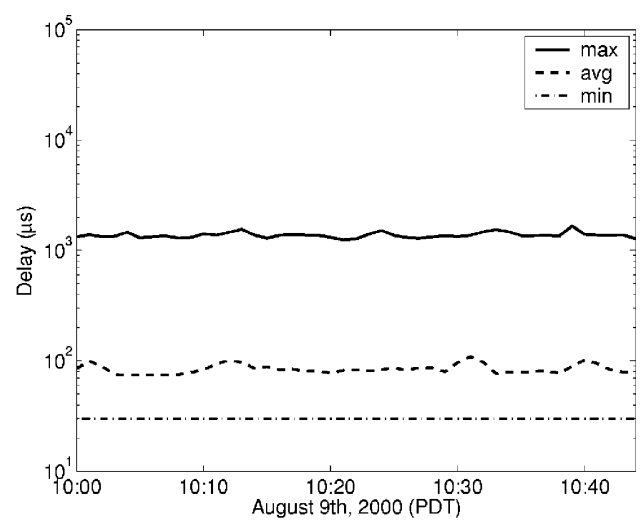

(b)

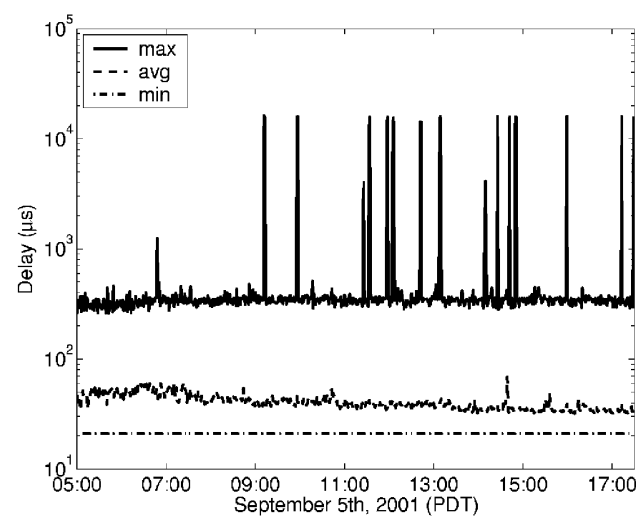

(d)

Fig. 2. Minimum, average, and maximum delay per minute for the matched packets of all four data sets. The $x$ axis is adjusted to the duration of the data set. The $y$ axis spans between $10 \mu \mathrm{s}$ and $100 \mathrm{~ms}$ for set 1 , set2, and set4. For set 3 the $y$ axis spans between $1 \mu \mathrm{s}$ and $10 \mathrm{~ms}$, given that delays are much lower than in the other three data sets. (a) set1 (OC-3). (b) set2 (OC-3). (c) set3 (OC-12), (d) set4 (OC-12).

the output link: $l_{m} / C_{\text {out }}$, where $l_{m}$ is the length of the $m$ th matched packet, and $C_{\text {out }}$ is the output link capacity. ${ }^{1}$ We refer to the difference between the total delay of packet $m$ and its transmission time on the output link as the router transit time, denoted by $d_{t x}^{-}(m): d_{t x}^{-}(m)=d(m)-l_{m} / C_{\text {out }}$. The empirical probability density function of $d_{t x}^{-}(m)$ is plotted in Fig. 4.

There still are three distinct peaks in the distribution, even though they are less pronounced than in Fig. 3. This may indicate that there is still a part of the router transit time that depends on the packet size.

2) Minimum Router Transit Time: When a packet arrives at a router, its destination address is looked up in the forwarding table, the appropriate output port is determined, and the packet is then transferred to the output port. Routers in our network do store-and-forward, as opposed to cut through [12]. This operation imposes a minimum amount of delay on every packet, proportional to its size, which is likely to explain those remaining peaks in Fig. 4. Below we quantify the minimum router transit time experienced by packets in our data sets.

We plot the minimum value of the router transit time for each packet size $L, d_{\min }(L)$, in Fig. 5

$$
d_{\min }(L)=\min _{1 \leq m \leq M}\left\{d_{t x}^{-}(m) \mid l_{m}=L\right\}
$$

${ }^{1}$ Throughout this paper, for the OC- 3 traces we set $C_{\text {out }}=150.336 \mathrm{Mb} / \mathrm{s}$, which is the effective payload of POS OC-3. For the OC-12 traces $C_{\text {out }}=$ $601.344 \mathrm{Mb} / \mathrm{s}$
Fig. 5 indicates that there exists a linear relationship between the two metrics. This relationship is made explicit through a linear regression. Given that all data sets feature an order of magnitude more packets for the size of 40, 576, and 1500 bytes, those three packet sizes are more likely to provide us with accurate minimum values for the router transit time. For this reason, we rely on the measurements for these three packet sizes in linear regression, and obtain (1)

$$
d_{\min }(L)=\left\{\begin{array}{ll}
0.0213 \cdot L+25, & \text { for set1/set2 } \\
0.0089 \cdot L+7, & \text { for set3 } \\
0.0192 \cdot L+18, & \text { for set4 }
\end{array} .\right.
$$

The linear relationship between minimum router transit time and packet size is consistent for the OC-3 data sets, and differs for the OC-12 data sets. We notice that for set 3 and set 4 we need two different equations to express the relationship between the minimum router transit time and the packet size. The reason for that is that packets that are received and transmitted on the same linecard exhibit a different behavior compared with the packets that need to transit the switch fabric. From (1), we can identify the effect that such features of the router architecture may have on the packet delay.

According to (1), transmission of packets across different linecards for OC-3 and OC-12 rates (set1, set2, and set4) leads to similar values for the slope capturing the linear relationship between packet size and minimum router transit time. 


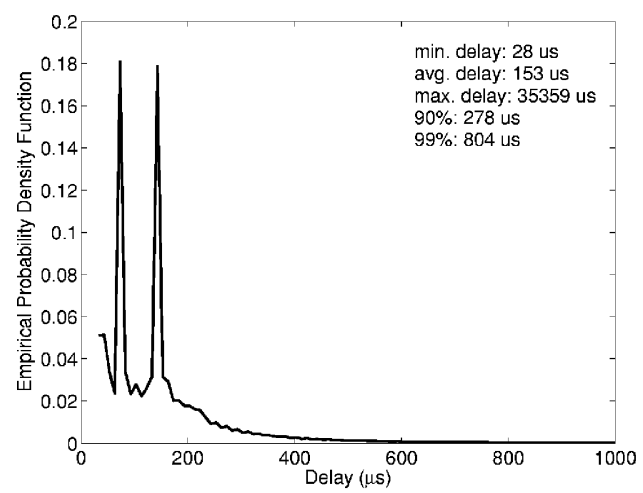

(a)

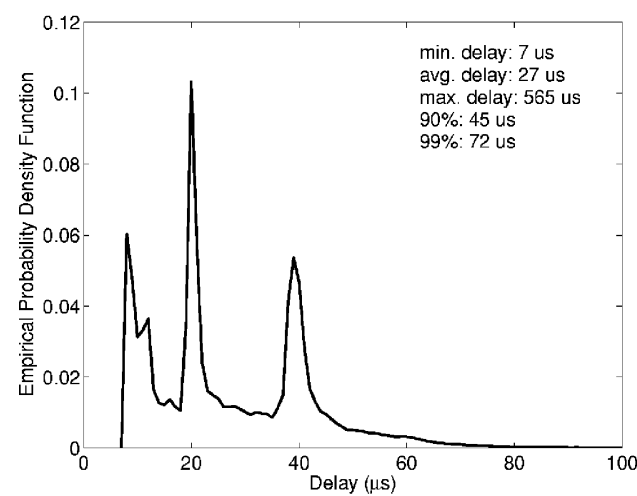

(c)

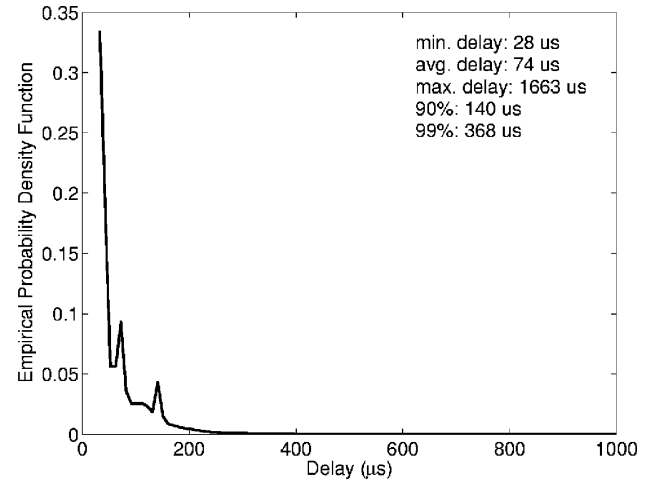

(b)

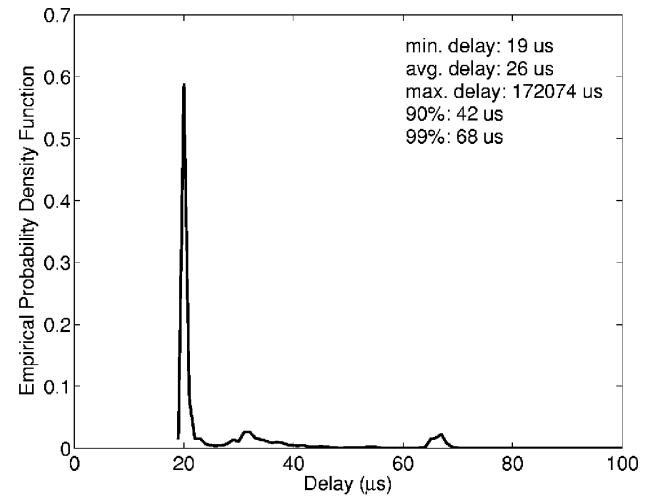

(d)

Fig. 3. Empirical probability density function of delay of matched packets $\{d(m)\}$. (a) set1 (OC-3). (b) set2 (OC-3). (c) set3 (OC-12). (d) set4 (OC-12).

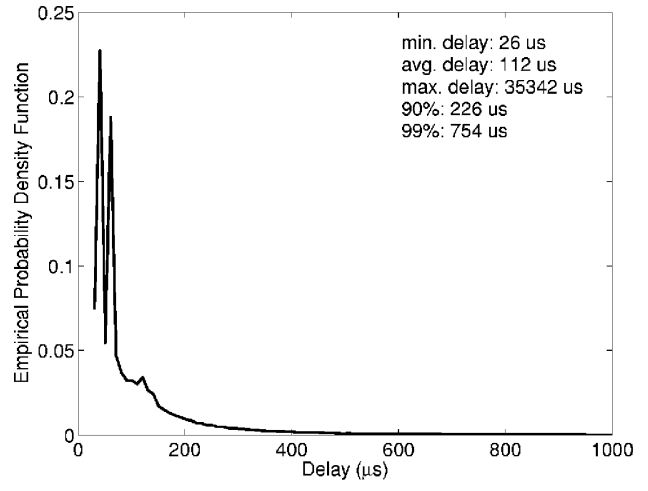

(a)

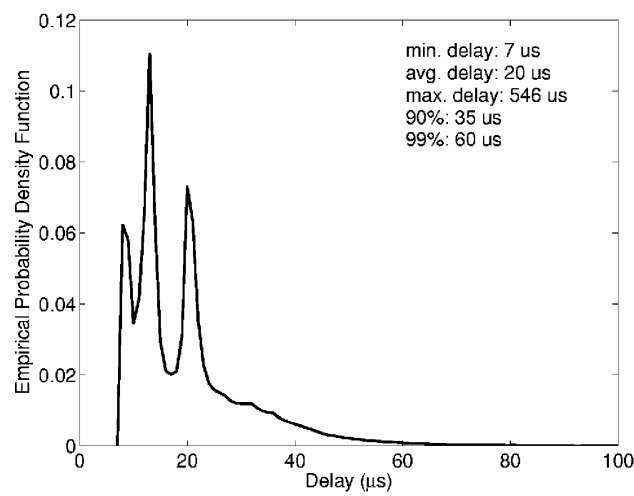

(c)

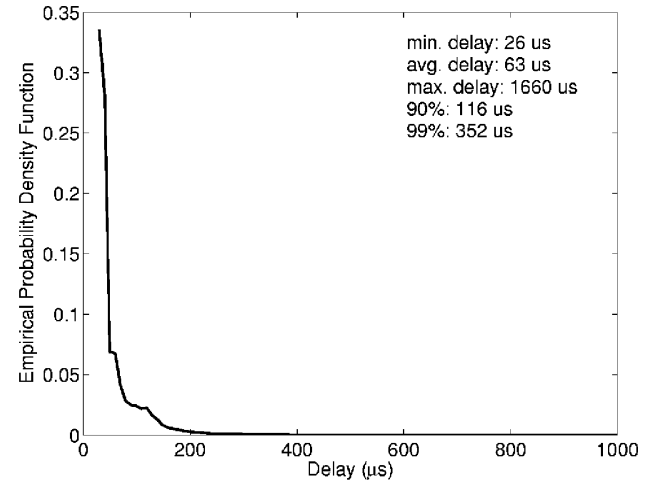

(b)

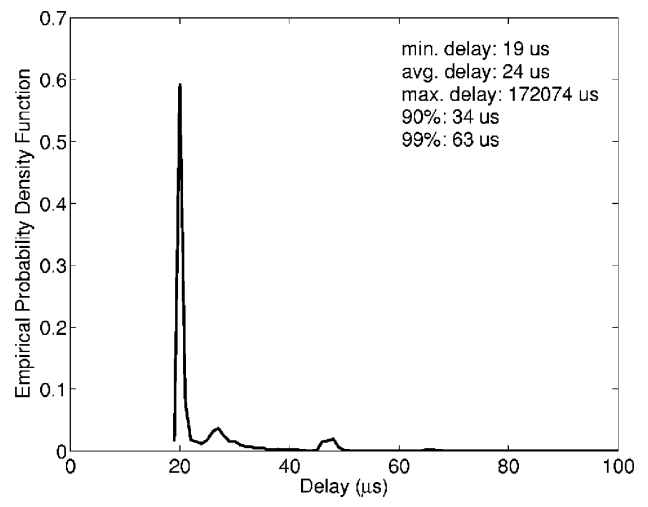

(d)

Fig. 4. Empirical probability density function of router transit time $d_{t x}^{-}(m)=d(m)-l_{m} / C_{\text {out }}$. (a) set1 (OC-3). (b) set2 (OC-3). (c) set3 (OC-12). (d) set4 (OC-12). 


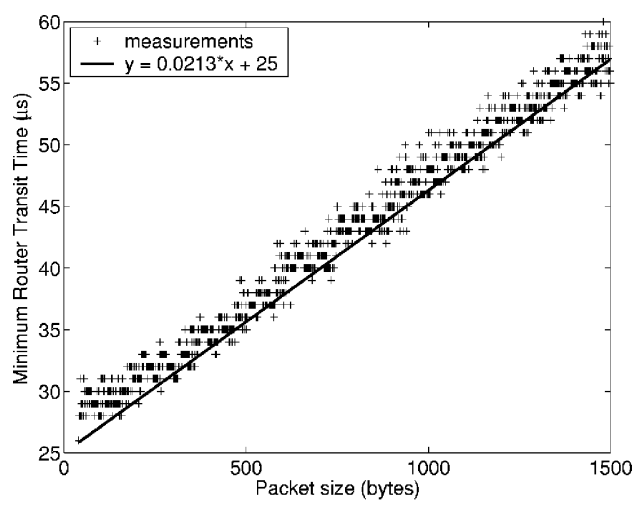

(a)

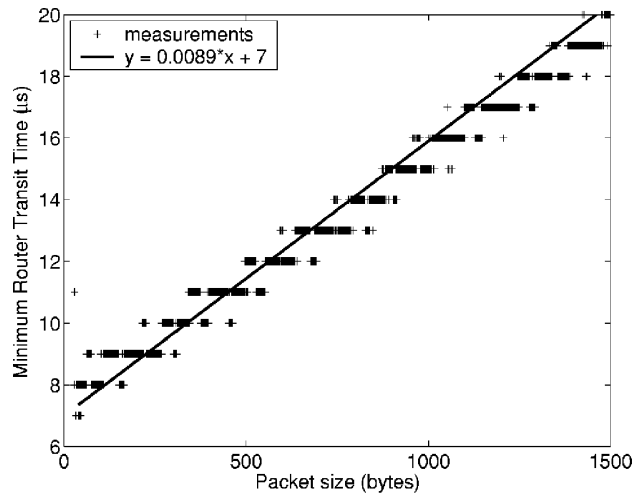

(c)

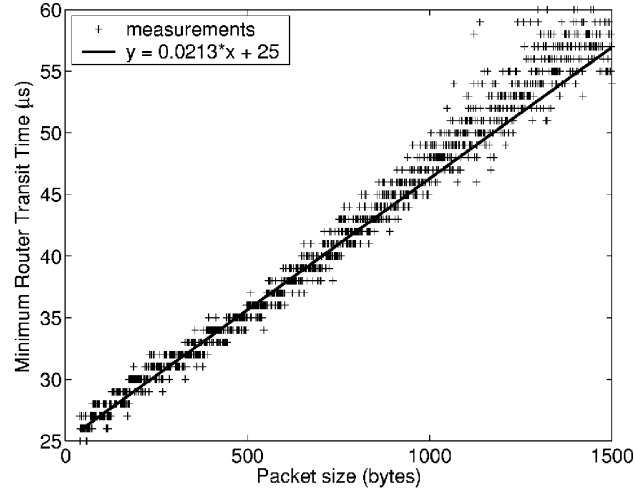

(b)

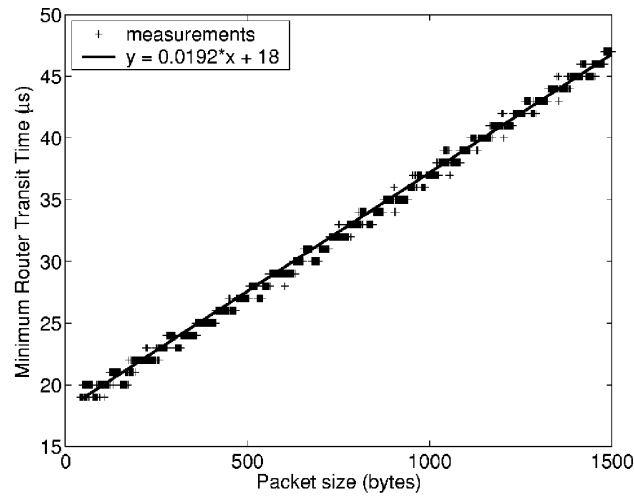

(c)

Fig. 5. Minimum router transit time versus packet size $L: \min _{m} d_{t x}^{-}(m)$ for $m \in\left\{i \mid l_{i}=L\right\}$. Each figure contains one value, for the minimum router transit time, for each packet size observed in our data. Delay granularity is $1 \mu \mathrm{s}$. (a) set1 (OC-3). (b) set2 (OC-3). (c) set3 (OC-12). (d) set4 (OC-12).

However, the constant term is different and larger for the OC-3 case. This could be attributed to the fact that set 4 does not involve two quad linecards (Table II), or that OC-12 linecards utilize more recent technology and, thus, may be offering faster packet processing than OC-3 linecards.

One important result derived from (1) and Fig. 5 is that packets which remain in the same OC-12 linecard are served much faster, i.e., in 7-20 $\mu$ s. Packets that have to be transmitted across the switch fabric are served in 19-39 $\mu$ s. Similar analysis performed on other data sets containing packets received and transmitted in the same quad linecard or across different linecards led to results consistent with this finding.

Subtracting $d_{\min }\left(l_{m}\right)$ from the router transit time, $d_{t x}^{-}(m)$, we obtain the actual amount of time packets have to wait inside the router. The new empirical probability density function is presented in Fig. 6 .

Packet size related peaks have now disappeared and the delay distributions look similar for all data sets. The distribution is characterized by very low delays: $45 \%$ of the packets in set 1 and more than $50 \%$ of the packets in set2 experience zero queueing delay. For the OC-12 data sets, almost $30 \%$ of the packets in set3 and $70 \%$ of the packets in set 4 go through the router without any queueing at the output link. Differences in the average delay can be explained by the packet size distribution of the data sets: set 1 and set 3 are dominated by packets larger than 500 bytes, while set 2 and set 4 contain mostly 40 byte packets. In addition, set 1 and set 3 consist of highly utilized links, thus featuring higher queueing delay values than set2 and set4. Small peaks around $100 \mu$ s for the OC-3 data sets and $20 \mu$ s for the OC-12 data sets correspond to the transmission of a maximum sized packet at the respective line rate; thus accounting for the fact that a packet may arrive at the output queue and find it occupied by another, possibly maximum-sized, packet. The 99th percentile delays are very small; below $750 \mu \mathrm{s}$ for the OC-3 data sets, and below $50 \mu$ s for the OC-12 data sets. Nevertheless, the maximum delay still reaches $172 \mathrm{~ms}$ for set 4 and $35 \mathrm{~ms}$ for set 1 .

\section{Possible Causes for Very Large Delay}

In Fig. 7, we present the cumulative distribution function (CDF) for the queueing delay $\left(\left(d_{t x}^{-}(m)-d_{\min }\left(l_{m}\right)\right)\right)$ observed for all four data sets. A key observation is that across all data sets the tail of the delay distribution is very long, accounting for the presence of very large delays. However, an examination of the output link data when the very large delays were observed shows that the link was not fully utilized while those packets were waiting. Therefore, part of the long delays is not due to queueing at the output link. In the remainder of this section, we look into possible explanations for these large delay values.

One possible reason could be that the monitoring systems lose synchronization. We exclude measurement equipment fault as a cause for large delays for the following reasons. If the two measurement systems had gone out of time synchronization, the minimum and average delay in Fig. 2 would exhibit a level shift over time, which is not visible. There is no way to tell if the system's software had a bug and produced the very large delays. However, it is extremely unlikely that a software bug 


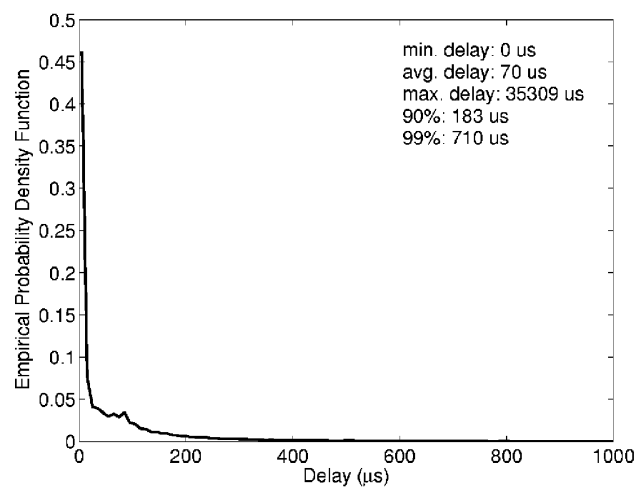

(a)

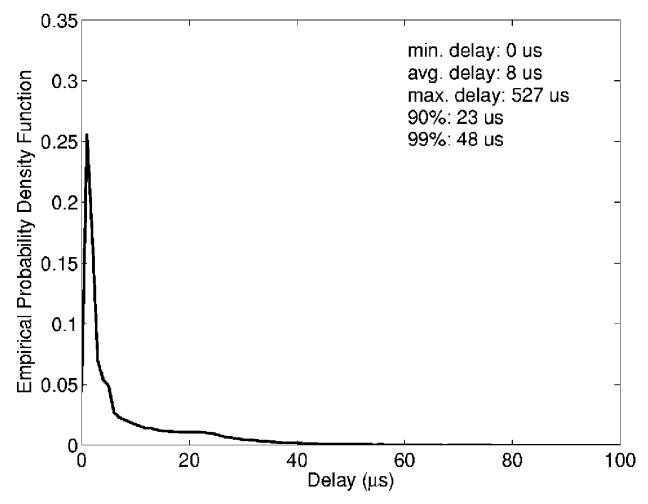

(c)

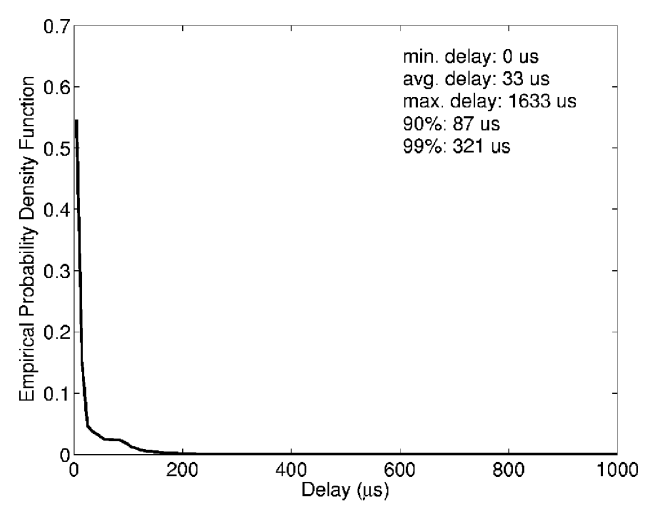

(b)

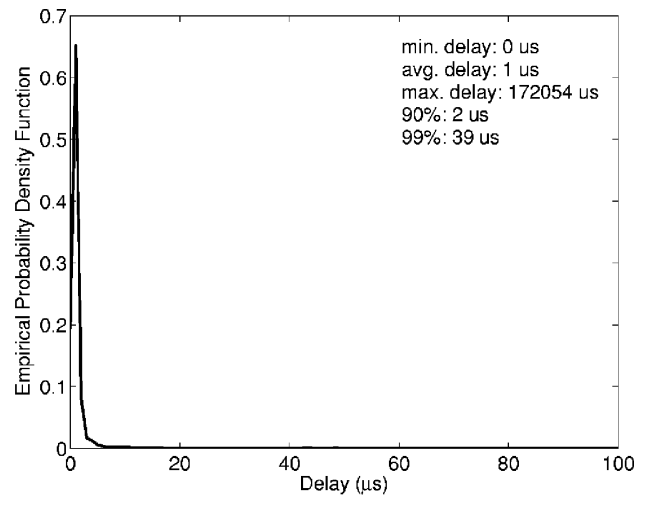

(d)

Fig. 6. Empirical probability density function of $\left(d_{t x}^{-}(m)-d_{\min }\left(l_{m}\right)\right)$. (a) set1 (OC-3). (b) set2 (OC-3). (c) set3 (OC-12), (d) set4 (OC-12).

affected only a handful of packets, still maintaining the strictly increasing nature of timestamps and keeping the minimum packet delay constant, both of which we checked in our traces.

A second reason, that can be easily verified, is that the packets experiencing long delays contain IP options. Most routers are designed to optimize the performance for the majority of packets. IP packets with options require additional processing in the protocol stack, and travel through a slower software path than packets without options. IP option packets are present in set2, set3, and set4. In Table III, we include the main statistics of the delay distribution derived from packets carrying IP options. Results indicate that packets with IP options spend at least $36 \mu$ s inside the router, and they usually account for the maximum delay in our single-hop delay distributions. The derived statistics should only serve as an indication for the magnitude of delay that packets with IP options may face while traversing a router, since the observed sample is too small to allow for generalization. Given that delay measurements for packets carrying IP options are not solely due to queueing, we do not include them in the remainder of our analysis.

Once IP option packets have been removed from our data sets, we find that the maximum delay for set 2 and set 4 drops significantly (Table IV). Due to the fact that there is a very small number of IP option packets present in our measurements, none of the other statistics of the distribution have significantly changed. Packets carrying IP options are capable of justifying the maximum delay in our data sets, but even after their removal the maximum delay experienced by packets in set 1 and set4 remains in the order of tens of milliseconds. Other potential reasons behind the very large delay values are: 1) routers stopping forwarding packets for a short period of time when busy with some other CPU intensive task, (e.g., routing table updates, SNMP requests, and garbage collection in memory), an effect usually referred to as a "coffee break;" 2) router interface cards with multiple ports or backplane switch fabrics that could allow input or output blocking [13]; and 3) memory locks or poor scheduling, etc.

\section{Filtering Based on a Single Output Queue Model}

When packets arrive at a router, they contend for resources to be forwarded to the destination output interface. The router can use various policies to resolve this contention. The first-in first-out (FIFO) output queue model captures the essence of how a router should serve packets contending for the same resource in a best-effort fashion [12]. Thus, we model an output port of a router as a single output queue. While a single output queue is not an accurate model of all the operations performed in the router, it is sufficient to allow us to determine if the delay of a packet is due to output queueing or not, using only the measurements we have at our disposal.

In the routers deployed in our network, packet processing is heavily pipelined so that a packet experiencing the minimum router transit time should not introduce extra queueing for the next packet arriving at the input port. That is, the minimum router transit time simply delays a packet's arrival time at the output queue, without affecting other packets. We can, thus, 


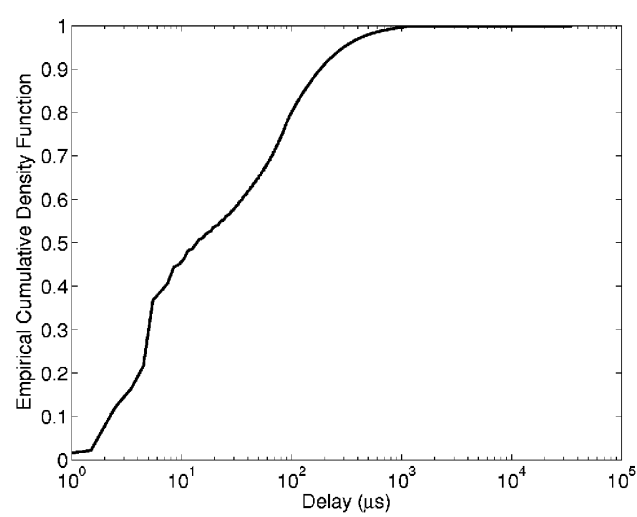

(a)

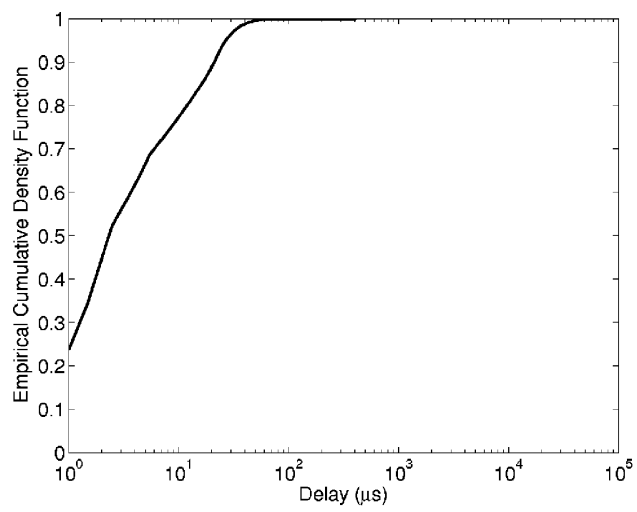

(c)

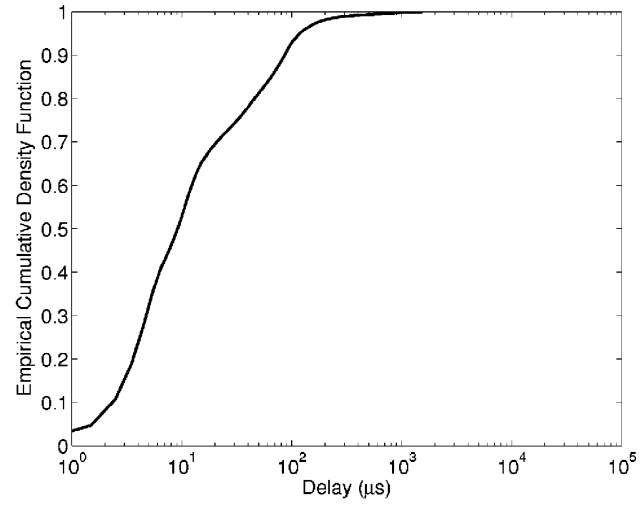

(b)

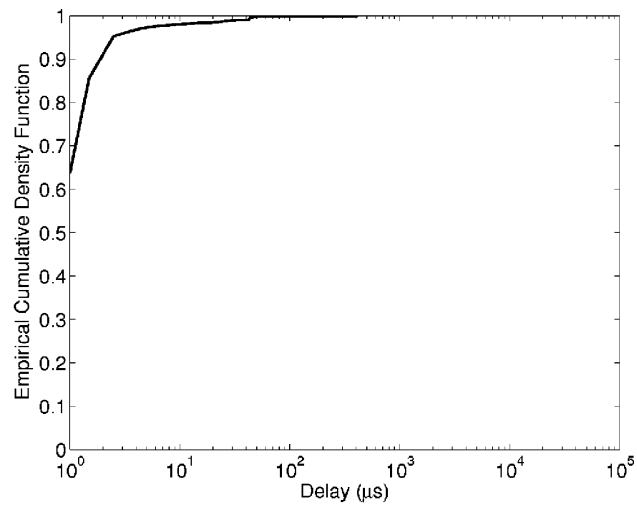

(d)

Fig. 7. Empirical cumulative density function of $\left(d_{t x}^{-}(m)-d_{\min }\left(l_{m}\right)\right)$. (a) set1 (OC-3). (b) set2 (OC-3). (c) set3 (OC-12). (d) set4 (OC-12).

TABLE III

DELAY STATISTICS FOR THE PACKETS THAT CARRY IP OPTIONS

\begin{tabular}{l|cccc}
\hline \multirow{2}{*}{$(\mu \mathrm{s})$} & \multicolumn{4}{|c}{ set2 } \\
\cline { 2 - 5 } & minimum & average & median & maximum \\
\hline$d_{t x}^{-}(m)$ & 242 & 453 & 307 & 1,659 \\
\hline \hline & \multicolumn{4}{|c}{ set 3 } \\
\multirow{2}{*}{$(\mu \mathrm{s})$} & minimum & average & median & maximum \\
\cline { 2 - 5 } & \multicolumn{4}{|c}{ matches } \\
\hline$d_{t x}^{-}(m)$ & 36 & 225 & 273 & 438 \\
\hline \multirow{3}{*}{$(\mu \mathrm{s})$} & set 4 \\
\cline { 2 - 5 } & minimum & average & median & maximum \\
\hline$d_{t x}^{-}(m)$ & 270 & 11,219 & 320 & 172,074 \\
\hline \multicolumn{5}{c}{}
\end{tabular}

assume that the $m$ th packet arrives at the output queue at $T_{\text {in }}^{\prime}(m)=T_{\text {in }}(m)+d_{\min }\left(l_{m}\right)$, and set the service rate of the single output queue to the transmission rate of the output link, as illustrated in Fig. 8.

We expect a packet to wait at the output queue if and only if the output queue is busy serving other packets. The waiting time of a packet is $T_{\text {out }}(m)-l_{m} / C_{\text {out }}-T_{\text {in }}^{\prime}(m)$. In Fig. 9, we plot the number of bytes transmitted at the output link out1 during the time interval of $\left[T_{\mathrm{in}}^{\prime}(m), T_{\text {out }}(m)-l_{m} / C_{\text {out }}\right]$ versus the size of the interval for set $1 .{ }^{2}$ The top line corresponds to the case when the number of bytes transmitted between the packet's arrival and

${ }^{2}$ Similar behavior is observed for the other three data sets and is omitted due to space limitations. departure time $\left[T_{\mathrm{in}}^{\prime}(m)\right.$ and $\left.T_{\text {out }}(m)-l_{m} / C_{\text {out }}\right]$ is equal to the number of bytes that would be transmitted if the link continuously sent packets at line rate. We observe that all data points lie below this top line. Moreover, most of the points that fall off this line are bounded by another line below, of the same slope, which allows for the transmission of one less maximum-sized packet. This latter line is described by $y=\left(C_{\text {out }} \cdot x\right) / 8-1500$, where $x$ is the size of the time interval in $\mu \mathrm{s}$, and $y$ is the number of bytes seen at the output link. We allow one maximum-sized packet as the error margin in our waiting time calculation, since the accuracy of the timestamps, the nonuniform distribution of SONET overhead in the signals, and the uncertainty about operations inside the router are likely to affect our computation. Those packets whose waiting times lie between the two lines are interpreted as follows: while a matched packet is waiting to be transmitted between $T_{\text {in }}^{\prime}(m)$ and $T_{\text {out }}(m)-l_{m} / C_{\text {out }}$, the output link is fully utilized. We consider as the filtered data set those packets that lie between the two bounding lines in Fig. 9. For set 1 the filtered set contains $94 \%$ of the total number of packets in the set. Other packets are considered to have experienced delay not due to output queueing ${ }^{3}$ beyond the error margin and are filtered out. To evaluate the magnitude of the delay values that get filtered out by our simple output queue model, we proceed as follows.

\footnotetext{
${ }^{3}$ Strictly speaking, transmission and propagation delays are not due to queueing as well. However, we limit the use of nonqueueing delay only to the delay experienced at the output queue.
} 
TABLE IV

Statistics For the OC-3 AND OC-12 Data Sets After the Removal of Packets With IP Options

\begin{tabular}{|c|c|c|c|c|c|c|c|c|c|c|}
\hline \multirow[b]{2}{*}{ inl to outl $(\mu \mathrm{s})$} & \multicolumn{5}{|c|}{$\begin{array}{c}\text { original set } \\
2,781,201 \text { matches }\end{array}$} & \multicolumn{5}{|c|}{$\begin{array}{l}\text { non IP-options set } \\
2,781,201 \text { matches }\end{array}$} \\
\hline & min. & avg. & $90 \%$ & $99 \%$ & max. & $\min$. & avg. & $90 \%$ & $99 \%$ & $\max$. \\
\hline$d_{t x}^{-}(m)$ & 26 & 112 & 226 & 754 & 35,342 & 26 & 112 & 226 & 754 & 35,342 \\
\hline$d_{t x}^{-}(m)-d_{\min }\left(l_{m}\right)$ & 0 & 70 & 183 & 710 & 35,309 & 0 & 70 & 183 & 710 & 35,309 \\
\hline \multirow[b]{2}{*}{ in 2 to out $2(\mu \mathrm{s})$} & \multicolumn{5}{|c|}{$\begin{array}{c}\text { original set } \\
1,175,665 \text { matches }\end{array}$} & \multicolumn{5}{|c|}{$\begin{array}{l}\text { non IP-options set } \\
1,175,656 \text { matches }\end{array}$} \\
\hline & min. & avg. & $90 \%$ & $99 \%$ & max. & min. & avg. & $90 \%$ & $99 \%$ & $\max$. \\
\hline$d_{t x}^{-}(m)$ & 26 & 63 & 116 & 352 & 1,660 & 26 & 63 & 116 & 352 & 1,547 \\
\hline$d_{t x}^{-}(m)-d_{\min }\left(l_{m}\right)$ & 0 & 33 & 87 & 321 & 1,633 & 0 & 33 & 87 & 321 & 1,520 \\
\hline \multirow[b]{2}{*}{ in 3 to out $3(\mu \mathrm{s})$} & \multicolumn{5}{|c|}{$\begin{array}{c}\text { original set } \\
17,613,183 \text { matches }\end{array}$} & \multicolumn{5}{|c|}{$\begin{array}{c}\text { non IP-options set } \\
17,613,162 \text { matches }\end{array}$} \\
\hline & min. & avg. & $90 \%$ & $99 \%$ & max. & min. & avg. & $90 \%$ & $99 \%$ & max. \\
\hline$\overline{d_{t x}^{-}(m)}$ & 7 & 20 & 35 & 60 & 546 & 7 & 20 & 35 & 60 & $\overline{546}$ \\
\hline$d_{t x}^{-}(m)-d_{\min }\left(l_{m}\right)$ & 0 & 8 & 23 & 48 & 527 & 0 & 8 & 23 & 48 & 527 \\
\hline \multirow[b]{2}{*}{ in4 to out4 $(\mu \mathrm{s})$} & \multicolumn{5}{|c|}{$\begin{array}{c}\text { original set } \\
70,423,140 \text { matches }\end{array}$} & \multicolumn{5}{|c|}{$\begin{array}{c}\text { non IP-options set } \\
70,423,101 \text { matches }\end{array}$} \\
\hline & min. & avg. & $90 \%$ & $99 \%$ & $\max$. & min. & avg. & $90 \%$ & $99 \%$ & $\max$. \\
\hline$d_{t x}^{-}(m)$ & 19 & 24 & 34 & 63 & 172,074 & 19 & 24 & 34 & 63 & 16,091 \\
\hline$d_{t x}^{-}(m)-d_{\min }\left(l_{m}\right)$ & 0 & 2 & 2 & 39 & 172,054 & 0 & 2 & 2 & 39 & 16,045 \\
\hline
\end{tabular}
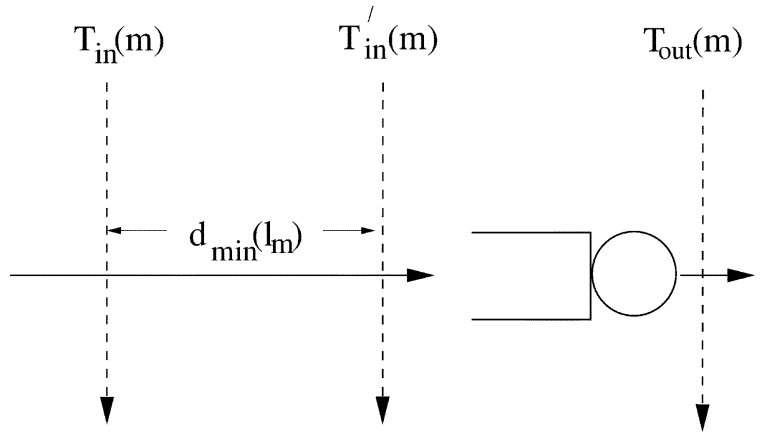

Fig. 8. Single output queue model of a router.

We compute the amount of delay that each packet should have experienced in all four data sets according to the observed output link utilization. We then subtract the computed delay value from the actual delay value measured. The difference between those two values corresponds to the amount of additional delay that a packet experienced inside the router. In Fig. 10(a) and (b), we present the empirical probability and cumulative density function for the difference in delay experienced by the set of packets that got filtered out.

Fig. 10(a) shows that the part of our delay measurements that cannot be explained according to our single output queue model may reach up to tens of milliseconds. An important observation is that for set 1 and set 2 the empirical probability density function shows a plateau between $10 \mu$ s and $1 \mathrm{~ms}$. For set4, the plateau area spans between 10-200 $\mu \mathrm{s}$. This behavior is consistent with a "coffee break" effect. When a router goes into a "coffee break," it stops forwarding packets for the duration of the break. Therefore, all packets that arrive at the router during this period have to wait until the router resumes the forwarding process and, therefore, experience delays that may reach the duration of the break. In our case, the observed behavior resembles a "coffee break" of $1 \mathrm{~ms}$ in our OC-3 measurements, and $200 \mu \mathrm{s}$ in our OC-12 measurements.

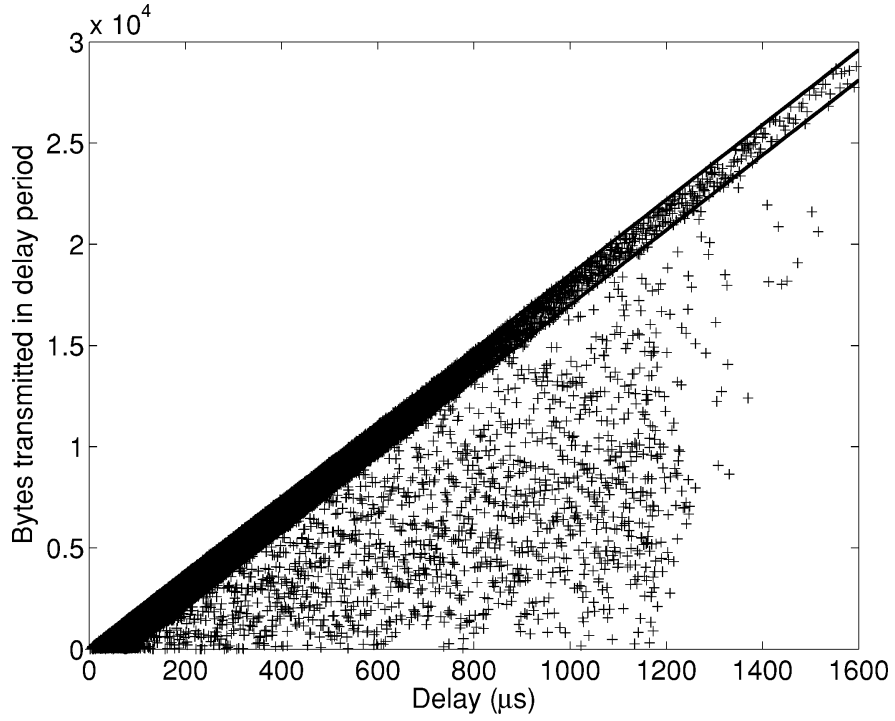

Fig. 9. Number of bytes transmitted between $T_{\text {in }}^{\prime}(m)$ and $T_{\text {out }}(m)-$ $l_{m} / C_{\text {out }}$ on out 1 .

What is interesting is that no such effect is evident for set3, where the maximum delay difference is limited to $17 \mu \mathrm{s}$. Recall that set3 corresponds to delay measurements taken inside the same quad-OC-12 linecard. Therefore, such a finding could be an indication that the "coffee break" effect does not take place at the linecards themselves. Unfortunately, seeking explanation for such a phenomenon requires detailed knowledge of the router architecture, which constitutes proprietary information. Therefore, we can only conjecture about possible reasons behind this behavior. Justification for the existence of delay discrepancies larger than $1 \mathrm{~ms}$ is even harder to provide. Queueing taking place at the input link and contention for access to the fabric switch could be possible explanations, but cannot be verified solely based on our measurements. In any case, as can be seen from Fig. 10(b), such a phenomenon affects 


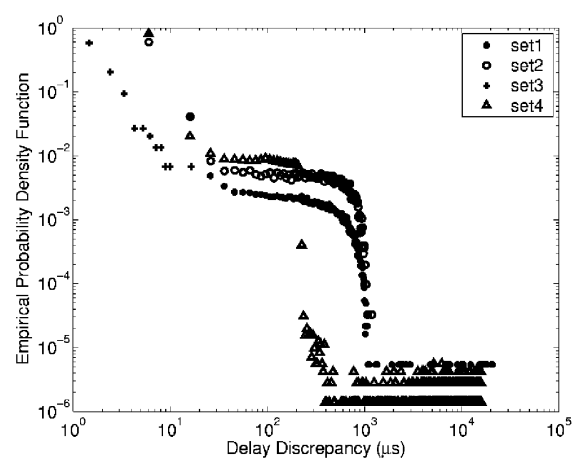

(a)

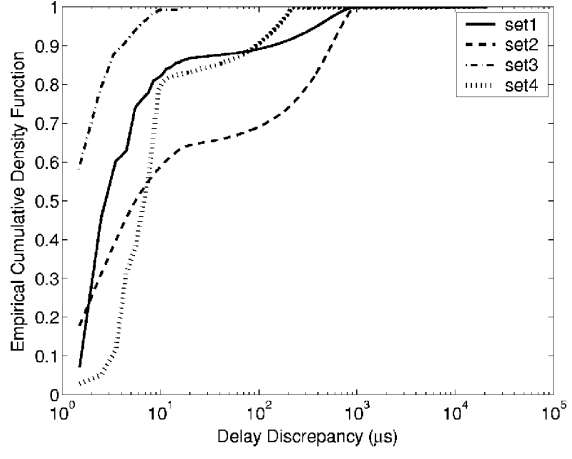

(b)

Fig. 10. Unexplained part of the delay in the measurements that get filtered out. (a) Empirical probability density function (log-log). (b) Empirical cumulative density function (log-normal).

TABLE V

Statistics For the OC-3 AND OC-12 Data SETs Before AND After Filtering

\begin{tabular}{|c|c|c|c|c|c|c|c|c|c|c|}
\hline \multirow[b]{2}{*}{ inl to outl $(\mu \mathrm{s})$} & \multicolumn{5}{|c|}{$\begin{array}{c}\text { original set } \\
2,781,201 \text { matches }\end{array}$} & \multicolumn{5}{|c|}{$\begin{array}{c}\text { filtered set } \\
2,596,486 \text { matches } \\
\text { (6.6\% filtered out) }\end{array}$} \\
\hline & min. & avg. & $90 \%$ & $99 \%$ & $\max$. & min. & avg. & $90 \%$ & $99 \%$ & $\max$ \\
\hline$d_{t_{x}}^{-}(m)$ & 26 & 112 & 226 & 754 & 35,342 & 26 & 106 & 217 & 603 & 3,937 \\
\hline$d_{t x}^{-}(m)-d_{\min }\left(l_{m}\right)$ & 0 & 70 & 183 & 710 & 35,309 & 0 & 65 & 174 & 558 & 3,903 \\
\hline \multirow[b]{2}{*}{ in 2 to out $2(\mu \mathrm{s})$} & \multicolumn{5}{|c|}{$\begin{array}{l}\text { non IP-options set } \\
1,175,665 \text { matches }\end{array}$} & \multicolumn{5}{|c|}{$\begin{array}{c}\text { filtered set } \\
1,145,170 \text { matches } \\
\text { (2.5\% filtered out) }\end{array}$} \\
\hline & min. & avg. & $90 \%$ & $99 \%$ & $\max$. & min. & avg. & $90 \%$ & $99 \%$ & $\max$ \\
\hline$d_{t x}^{-}(m)$ & 26 & 63 & 116 & 352 & 1,547 & 26 & 56 & 112 & 230 & 1,547 \\
\hline$d_{t x}^{-}(m)-d_{\min }\left(l_{m}\right)$ & 0 & 33 & 87 & 321 & 1,520 & 0 & 27 & 82 & 200 & 1,520 \\
\hline \multirow[b]{2}{*}{ in 3 to $o u t 3(\mu \mathrm{s})$} & \multicolumn{5}{|c|}{$\begin{array}{l}\text { non IP-options set } \\
17,613,183 \text { matches }\end{array}$} & \multicolumn{5}{|c|}{$\begin{array}{c}\text { filtered set } \\
17,613,018 \text { matches } \\
(0.0009 \% \text { filtered out })\end{array}$} \\
\hline & min. & avg. & $90 \%$ & $99 \%$ & max. & min. & avg. & $90 \%$ & $99 \%$ & $\max$ \\
\hline$d_{t x}^{-}(m)$ & 7 & 20 & 35 & 60 & 546 & 7 & 20 & 35 & 60 & 546 \\
\hline$d_{t x}^{-}(m)-d_{\min }\left(l_{m}\right)$ & 0 & 8 & 23 & 48 & 527 & 0 & 8 & 23 & 48 & 527 \\
\hline \multirow[b]{2}{*}{ in4 to out4 ( $\mu \mathrm{s})$} & \multicolumn{5}{|c|}{$\begin{array}{c}\text { non IP-options set } \\
70,423,140 \text { matches }\end{array}$} & \multicolumn{5}{|c|}{$\begin{array}{c}\text { filtered set } \\
69,710,887 \text { matches } \\
\text { (1\% filtered out) }\end{array}$} \\
\hline & min. & avg. & $90 \%$ & $99 \%$ & max. & min. & avg. & $90 \%$ & $99 \%$ & $\max$ \\
\hline$d_{t x}^{-}(m)$ & 19 & 24 & 34 & 63 & 16,091 & 19 & 24 & 33 & 49 & 362 \\
\hline$d_{t x}^{-}(m)-d_{\min }\left(l_{m}\right)$ & 0 & 2 & 2 & 39 & 16,045 & 0 & 1 & 2 & 16 & 160 \\
\hline
\end{tabular}

a very small number of packets, namely between $20 \%-40 \%$ of the filtered out packets. This percentage corresponds to less than $1 \%$ of the total number of packets in each data set. ${ }^{4}$

Given that delays experienced by packets beyond our error margins are not related to queueing at the output link, we continue our analysis only with the filtered data sets. We summarize the statistics for the router transit time and queueing delay for the original and filtered data sets in Table V. The average, 90th, and 99th percentile values of the delay distribution are all lower for the filtered data sets. Moreover, all of the delays larger than $5 \mathrm{~ms}$ in set 1 and set 4 have disappeared, and the maximum delay has dropped to $3.9 \mathrm{~ms}$ and $160 \mu \mathrm{s}$, respectively. On the other hand, the maximum delay for set 2 and set 3 remains unchanged, indicating that the output link was fully utilized when the maximally delayed packet was being held back from transmission. We plot the minimum, average, and maximum values

${ }^{4}$ The final percentage of packets that get filtered out is higher than $1 \%$ because of small delay discrepancies, below $10 \mu \mathrm{s}$. of the filtered delays for all four data sets in Fig. 11. We compare with Fig. 2 and notice that the maximum delay does not stay over $1 \mathrm{~ms}$ throughout the entire day. Consequently, the single queue model is effective in filtering the delays which are not due to queueing at the output link.

\section{Queuing Delay TAIL BehaVior}

In this section, we analyze the tail of the queueing delay distribution. This analysis will help us identify possible models for the queueing delay in the backbone that could be exploited in simulation environments. We show that our results agree with previous analytical findings described in [2].

Tail behavior can be categorized into three types: light tailed, long tailed, and heavy tailed. A light tailed distribution has a probability density function whose tail approaches zero at least as rapidly as an exponential distribution. A distribution is said to have a heavy tail if $P[X>x] \sim k x^{-a}$ as $x \rightarrow \infty, 0<$ 


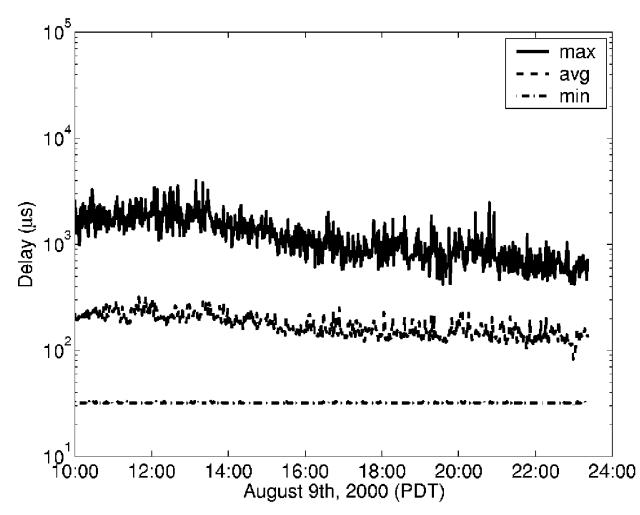

(a)

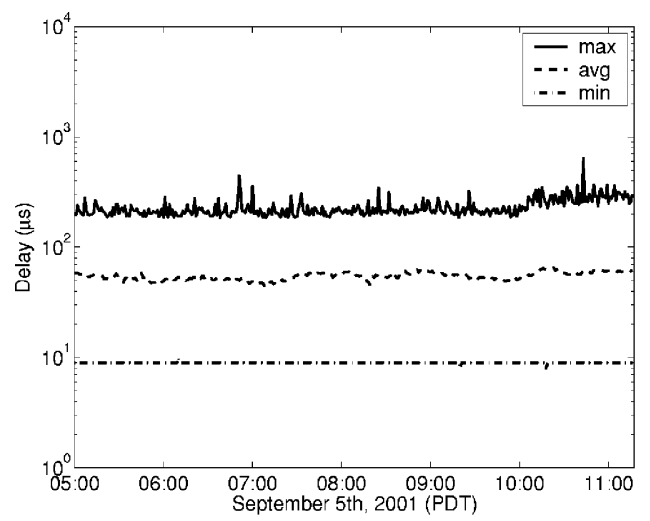

(c)

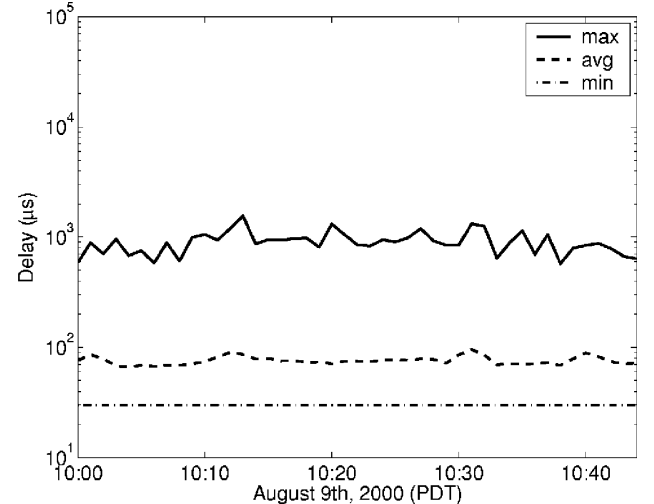

(b)

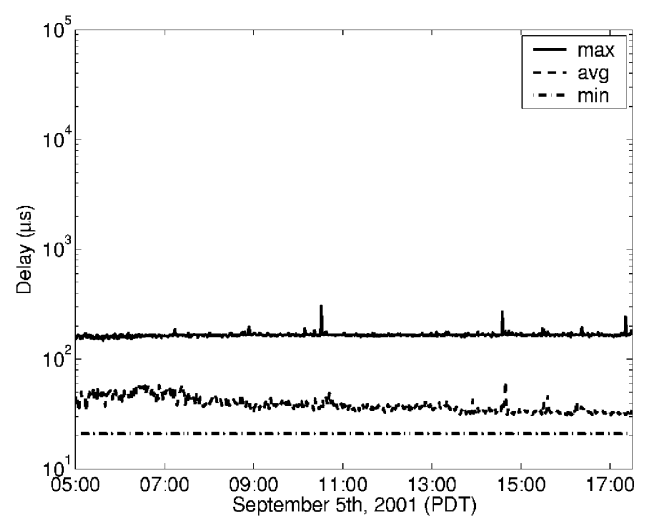

(d)

Fig. 11. Minimum, average, and maximum single-hop delays per minute for the filtered packets of all four data sets. The $x$ axis is adjusted to the duration of the data set. The $y$ axis spans between $10 \mu \mathrm{s}$ and $100 \mathrm{~ms}$ for set 1 , set2, and set 4 . For set 3 the $y$ axis spans between $1 \mu \mathrm{s}$ and $10 \mathrm{~ms}$, given that delays are much lower than in the other three data sets. (a) set1 (OC-3). (b) set2 (OC-3). (c) set 3 (OC-12). (d) set4 (OC-12).

$a<2$ [14]. This means that regardless of the distribution for small values of the random variable, if the asymptotic shape of the distribution is hyperbolic, the distribution is heavy tailed. The simplest heavy tailed distribution is the Pareto distribution which is hyperbolic over its entire range and has a probability mass function $p(x)=a k^{a} x^{-a-1}, a, k>0, x \geq k$, where $k$ represents the smallest value the random variable can take. Long tailed distributions decay slower than an exponential, without being heavy tailed. Lognormal and Weibull ${ }^{5}$ distributions with the shape parameter $b<1$ belong to long tailed distributions.

The network traffic is known to be long-range dependent, and such traffic can be modeled as FBM [4]. Norros shows that the queueing delay distribution of the FBM traffic is approximated by a Weibull distribution [2].

To examine what tail category our delay distributions fall into, we first plot the complementary cumulative distribution function $(\mathrm{CCDF})$ of $\left(d_{t x}^{-}(m)-d_{\min }\left(l_{m}\right)\right)$ in $\log -\log$ scale for the first hour of the filtered sets, where link utilization remains approximately constant (Fig. 12). If the tail of the CCDF forms a straight line, then the distribution may be heavy tailed. From Fig. 12, it is not clear whether this is the case for our data sets. We use the aest tool to formally check if the queueing delay distribution is heavy tailed [15]. The obtained results indicate

\footnotetext{
${ }^{5}$ The probability density function of a Weibull distribution is given by $f(x)=$ $\left(b x^{b-1} / a^{b}\right) e^{-(x / a)^{b}}$, with $a>0, b>0 ; \alpha$ is called the scale parameter, while $b$ is called the shape parameter.
}

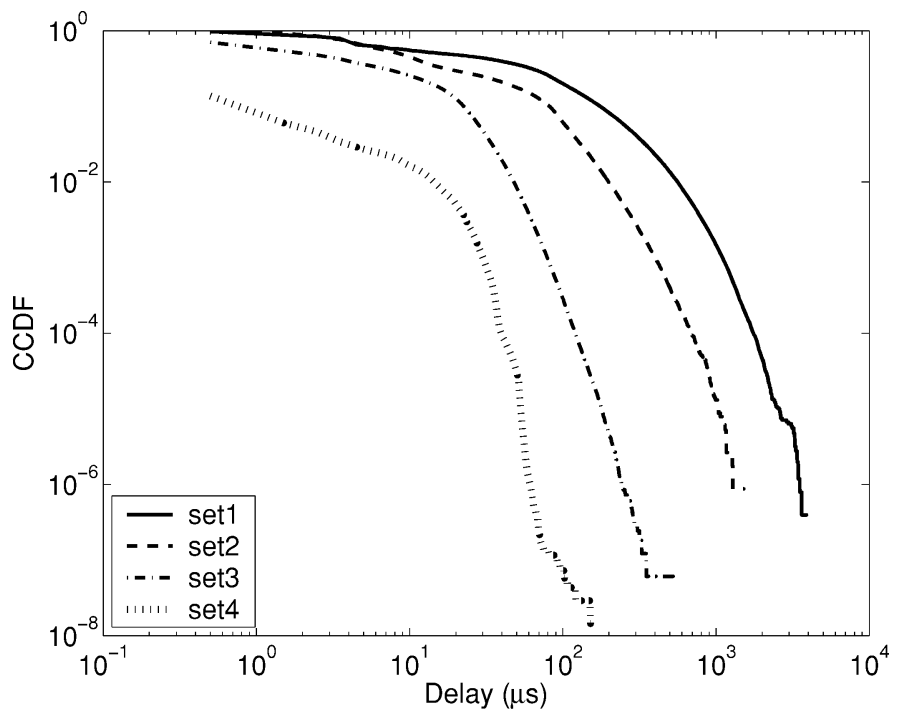

Fig. 12. $\log -\log$ plot of CCDF for the queueing delay measured for all four data sets (data set 1, 2: OC-3, data set 3, 4: OC-12).

that our delay distributions do not have the power-law tail like the Pareto distribution, and are not heavy tailed.

We then look into whether our queueing delay distributions are long-tailed. As already mentioned, a Weibull distribution with a shape parameter $\beta$ less than one belongs to the long-tailed 


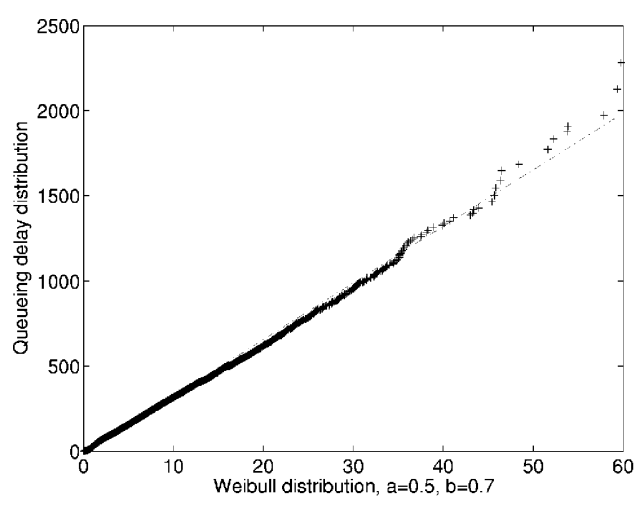

with $b=0.7$

(a)

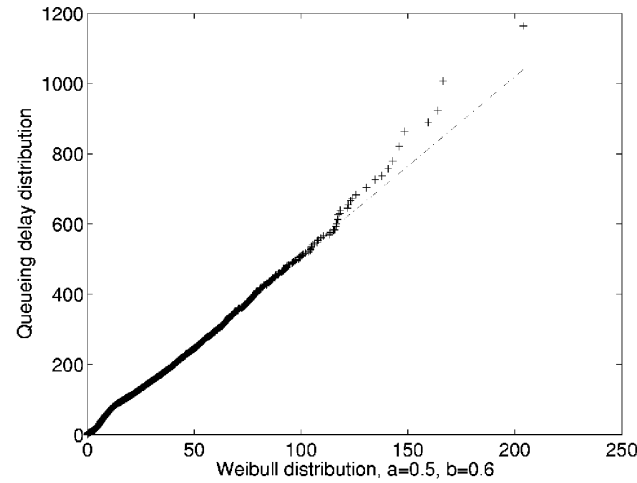

with $b=0.6$

(b)

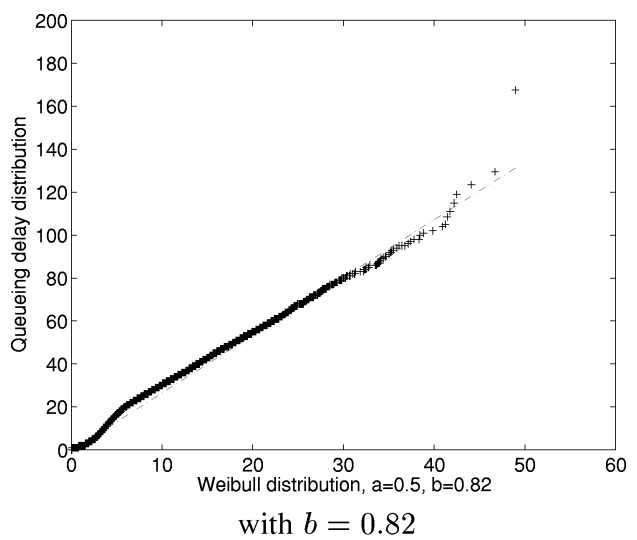

(c)

Fig. 13. Quantile-quantile plot of the queueing delay distribution against a Weibull distribution. (a) set1 (OC-3) with $b=0.7$. (b) set2 (OC-3) with $b=0.6$. (c) $\operatorname{set} 3(\mathrm{OC}-12)$ with $b=0.82$.

distributions. We fit a Weibull distribution to our queueing delay distributions, and present our results in Fig. 13 for the first three data sets. Set 4 is omitted because it is characterized by input and output link utilization of less than $10 \mathrm{Mb} / \mathrm{s}$ out of the $622 \mathrm{Mb} / \mathrm{s}$ of the link's capacity. As a consequence, the respective queueing delay distribution is characterized by a 99th percentile equal to $15 \mu \mathrm{s}$. This means that the number of samples we have in the tail of this particular distribution is very limited. Moreover, given their magnitude, the sample values are sensitive to our clock accuracy and our $1 \mu$ s granularity. Fig. 13 shows that the queueing delay distribution for set 1 and set2 fits to a Weibull distribution with a shape parameter $b$ equal to 0.6 and 0.7 , respectively. The OC-12 distribution for queueing delay inside the same linecard (set3) can be approximated with a Weibull distribution with a shape parameter $b$ equal to 0.82 . Therefore, the distribution of queueing delay is long tailed, confirming the finding in [2].

We further sort the three data sets in order of increasing output link utilization, i.e., set2, set1 and set3 (Table I). We notice that data sets characterized by higher output link utilization are also characterized by greater values of $b$ for their output queueing delay distribution. Thus, it appears that the output queueing delay distribution gets closer to an exponential distribution for higher output link utilizations. A similar finding was also reported in [16]. Nevertheless, further analysis is needed to confirm such a statement.

\section{CONCLUSION}

To the best of our knowledge, this work is the first to provide data about actual delays incurred through a single router in the backbone. We measure single-hop delay as experienced by packets in the Sprint IP backbone network. We develop a methodology to identify the contributing factors to the single-hop delay that is simple and applicable to any single-hop delay measurements. We demonstrate its applicability on OC-3 and $\mathrm{OC}-12$ packet traces. In addition to packet processing, transmission, and queueing delays, we identify the presence of very large delays that cannot be explained within the context of a work-conserving FIFO output queue. We provide a simple technique to remove these outliers from our measurements, and offer possible explanations regarding the events that may have led to such extreme delays through a single node.

According to our results, $99 \%$ of the packets in the backbone experience less than $1 \mathrm{~ms}$ of delay going through a single router when transmitted at OC-3 speeds. At OC-12 line rates, the single-hop delay drops to less than $100 \mu \mathrm{s}$. After the extraction of the queueing delay component in our measurements, we show that the largest part of single-hop delay experienced by a packet is not due to queueing, but rather to the processing and transmission of the packet across the switch fabric. In addition, we observe a small number of packets (less than $1 \%$ in our measurements) that may experience significantly larger delays, 
either because they carry IP options or because they are affected by idiosyncratic router behavior.

The analysis of the queueing delay distribution reveals that it can be approximated by a Weibull distribution with a scale parameter $a=0.5$, and a shape parameter $b=0.6 \sim 0.7$ for transmission of packets across two different OC-3 linecards. When packets are forwarded within the same linecard, i.e., they do not transit the switch fabric, and at OC-12 link speeds, the queueing delay distribution can be approximated with a Weibull distribution with a higher shape parameter $b=0.82$. Thus, the output queueing delay distribution is long-tailed confirming previous analytical findings by Norros [2]. We believe that identification and modeling of the several components comprising single-hop delay allow for more realistic backbone router models, that could easily be used in simulation environments.

In summary, packets in the Sprint IP backbone network experience edge-to-edge delays that are dominated by the propagation delay and face minimal jitter. This result, though, should be evaluated within the context of Sprint's backbone design principles that dictate moderate link utilization across the network; in our measurements all links were utilized less than $70 \%$ even at a $10-\mathrm{ms}$ time scale.

\section{ACKNOWLEDGMENT}

The authors would like to thank M. Crovella for providing us with the aest tool, J. Kurose, R. Cruz, J. Crowcroft, and S. Bhatti for their comments on the paper.

\section{REFERENCES}

[1] W. Leland, M. Taqqu, W. Willinger, and D. Wilson, "On the self-similar nature of Ethernet traffic," IEEE/ACM Trans. Networking, vol. 2, pp. 1-15, Feb. 1994.

[2] I. Norros, "On the use of fractional brownian motion in the theory of connectionless networks," IEEE J. Select. Areas Commun., vol. 13, pp. 953-962, Aug. 1995.

[3] A. Erramilli, O. Narayan, and W. Willinger, "Experimental queuing analysis with long-range dependent packet traffic," IEEE/ACM Trans. Networking, vol. 4, pp. 209-223, Apr. 1996.

[4] A. Feldmann, A. Gilbert, and W. Willinger, "Data networks as cascades: Investigating the multifractal nature of Internet WAN traffic," $A C M$ Comput. Commun. Rev., vol. 28, no. 4, pp. 42-55, Sept. 1998.

[5] A. Erramilli, O. Narayan, A. Neidhardt, and I. Saniee, "Performance impacts of multi-scaling in wide area TCP/IP traffic," in Proc. IEEE INFOCOM, Tel-Aviv, Israel, Mar. 2000, pp. 26-30.

[6] C. Fraleigh, C. Diot, B. Lyles, S. Moon, P. Owezarski, K. Papagiannaki, and F. Tobagi, "Design and deployment of a passive monitoring infrastructure," in Proc. Passive Active Measurement Workshop, Amsterdam, Apr. 2001.

[7] DAG 3.2 SONET network interface. [Online]. Available: http://dag.cs. waikato.ac.nz/dag/dag4-arch.html.

[8] K. Papagiannaki, S. Moon, C. Fraleigh, P. Thiran, F. Tobagi, and C. Diot, "Analysis of measured single-hop delay from an operational backbone network," in IEEE INFOCOM, New York, June 2002, pp. 535-544.

[9] D. Knuth, The Art of Computer Programming, Volume I: Fundamental Algorithms, 2nd ed. Reading, MA: Addison-Wesley, 1973.

[10] V. Paxson, "Measurements and analysis of end-to-end Internet dynamics," Ph.D. dissertation, Univ. California, Comput. Sci. Div., Berkeley, CA, 1997.

[11] K. Thompson, G. Miller, and R. Wilder, "Wide-area Internet traffic patterns and characteristics," IEEE Network, vol. 11, pp. 10-23, Nov./Dec. 1997.

[12] S. Keshav and S. Rosen, "Issues and trends in router design," IEEE Commun. Mag., vol. 36, pp. 144-151, May 1998.

[13] N. McKeown, "iSLIP: A scheduling algorithm for input-queued switches," IEEE Trans. Networking, vol. 7, pp. 188-201, Apr. 1999.
[14] D. Cox, "Long-range dependence: A review," in Statistics: An Appraisal, H. A. David and H. T. David, Eds. Ames, IA: Iowa State Univ. Press, 1984, pp. 55-74.

[15] M. Crovella and M. Taqqu, "Estimating the heavy tail index from scaling properties," Methodology and Computing in Applied Probability, vol. 1, no. 1, pp. 55-79, July 1999.

[16] J. Cao, W. Cleveland, D. Lin, and D. Sun, "Internet traffic tends toward Poisson and independent as the load increases," in Nonlinear Estimation and Classification, D. Denison, M. Hansen, C. Holmes, B. Mallick, and B. Yu, Eds. New York: Springer-Verlag, 2002, pp. 83-110

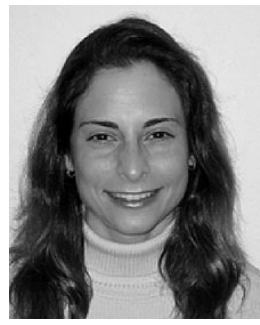

Konstantina Papagiannaki (S'02) received the B.S. degree in electrical and computer engineering from the National Technical University of Athens, Athens, Greece, in 1998, and the Ph.D. degree from the University College London, London, U.K., in 2003.

She has been a Member of the IP Research Group, Sprint Advanced Technology Laboratories, Burlingame, CA, since April 2000. Her research interests are in Internet measurements, modeling of Internet traffic, and backbone network traffic engineering.

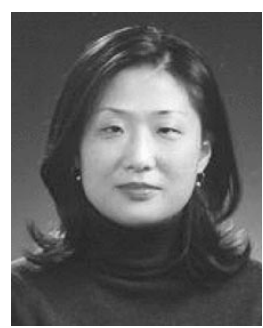

Sue Moon (S'86-A'00) received the B.S. and M.S. degrees from Seoul National University, Seoul, Korea, in 1988 and 1990, respectively, all in computer engineering, and the Ph.D. degree in computer science from the University of Massachusetts at Amherst.

From 1990 to 1991, she was with IMIGE Systems Inc., Seoul, Korea. Since 1999, she has been with Sprint Advanced Technology Laboratories, Burlingame, CA. Her main research interests are network performance measurement and monitoring, network delay, traffic scaling behavior analysis, and network anomalies.

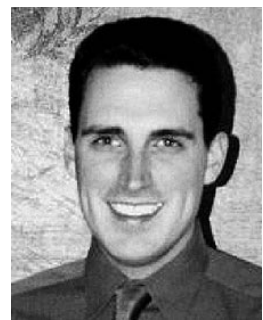

Chuck Fraleigh received the B.S. degree in computer and electrical engineering from Purdue University, West Lafayette, IN, in 1996, and the M.S. and Ph.D. degrees in electrical engineering from Stanford University, Stanford, CA, in 1998 and 2002, respectively.

From 1998 to 2002, he was a Student Visitor at Sprint Advanced Technology Laboratories, Burlingame, CA, and is now with NetVmg Inc., Fremont, CA. His research interests include Internet traffic measurement and modeling, network provisioning, and the design of network measurement systems.

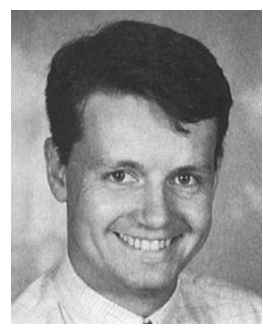

Patrick Thiran (S'88-M'90) received the electrical engineering degree from the Université Catholique de Louvain, Louvain-la-Neuve, Belgium, in 1989, the M.S. degree in electrical engineering from the University of California at Berkeley, in 1990, and the Ph.D. degree from the Swiss Federal Institute of Technology at Lausanne (EPFL), Lausanne, Switzerland, in 1996.

He became a Professor at EPFL in 1998, and was on leave with Sprint Advanced Technology Laboratories, Burlingame, CA, from 2000 to 2001. His research interests are in communication networks, performance analysis, dynamical systems, and stochastic models.

Dr. Thiran is a Fellow of the Belgian American Educational Foundation, and he received the 1996 EPFL Doctoral Prize. He served as an Associate Editor for the IEEE TRANSACTIONS ON CiRCUITS AND SYSTEMS, from 1997 to 1999. 


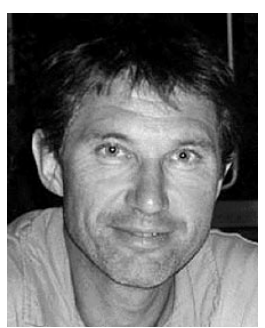

Christophe Diot received the Ph.D. degree in computer science from Institut National Polytechnique de Grenoble, Grenoble, France, in 1991.

From 1993 to 1998, he was a Research Scientist at Institut National de Rec herc he en Informatique et en Automatique (INRIA), Sophia Antipolis, France, working on new Internet architecture and protocols. He joined Sprint Advanced Technology Laboratories, Burlingame, CA, in October 1998 to take the lead of the IP Research Group. His current interest is in the passive monitoring of the Sprint IP backbone in order to study IP traffic characteristics and to design new analytical models and traffic engineering solutions for packet networks. 